\title{
Article \\ Protective Effect of Nebivolol against Oxidative Stress Induced by Aristolochic Acids in Endothelial Cells
}

\author{
Marie-Hélène Antoine ${ }^{1, * \mathbb{C}}$, Cécile Husson ${ }^{1}$, Tatiana Yankep ${ }^{1}$, Souhaila Mahria ${ }^{1}$, Vanessa Tagliatti ${ }^{2} \mathbb{D}$, \\ Jean-Marie Colet ${ }^{2}$ and Joëlle Nortier ${ }^{1}$ \\ 1 Laboratory of Experimental Nephrology, Faculty of Medicine, Université Libre de Bruxelles, Erasme Campus, \\ 808 Route de Lennik, B-1070 Brussels, Belgium; cecile.husson@ulb.be (C.H.); tatiana.Yankep@ulb.be (T.Y.); \\ souhaila.mahria@ulb.be (S.M.); joelle.nortier@ulb.be (J.N.) \\ 2 Laboratory of Human Toxicology, University of Mons (UMONS), 6 Avenue du Champ de Mars, \\ B-7000 Mons, Belgium; Vanessa.TAGLIATTI@umons.ac.be (V.T.); jean-marie.colet@umons.ac.be (J.-M.C.) \\ * Correspondence: marie-helene.antoine@ulb.be
}

check for updates

Citation: Antoine, M.-H.; Husson, C.; Yankep, T.; Mahria, S.; Tagliatti, V.; Colet, J.-M.; Nortier, J. Protective Effect of Nebivolol against Oxidative Stress Induced by Aristolochic Acids in Endothelial Cells. Toxins 2022, 14, 132. https://doi.org/10.3390/ toxins14020132

Received: 6 January 2022

Accepted: 7 February 2022

Published: 10 February 2022

Publisher's Note: MDPI stays neutral with regard to jurisdictional claims in published maps and institutional affiliations.

Copyright: (C) 2022 by the authors. Licensee MDPI, Basel, Switzerland. This article is an open access article distributed under the terms and conditions of the Creative Commons Attribution (CC BY) license (https:// creativecommons.org/licenses/by/ $4.0 /)$.
Abstract: Aristolochic acids (AAs) are powerful nephrotoxins that cause severe tubulointerstitial fibrosis. The biopsy-proven peritubular capillary rarefaction may worsen the progression of renal lesions via tissue hypoxia. As we previously observed the overproduction of reactive oxygen species (ROS) by cultured endothelial cells exposed to AA, we here investigated in vitro AA-induced metabolic changes by ${ }^{1} \mathrm{H}-\mathrm{NMR}$ spectroscopy on intracellular medium and cell extracts. We also tested the effects of nebivolol (NEB), a $\beta$-blocker agent exhibiting antioxidant properties. After $24 \mathrm{~h}$ of AA exposure, significantly reduced cell viability and intracellular ROS overproduction were observed in EAhy926 cells; both effects were counteracted by NEB pretreatment. After $48 \mathrm{~h}$ of exposure to AA, the most prominent metabolite changes were significant decreases in arginine, glutamate, glutamine and glutathione levels, along with a significant increase in the aspartate, glycerophosphocholine and UDP-N-acetylglucosamine contents. NEB pretreatment slightly inhibited the changes in glutathione and glycerophosphocholine. In the supernatants from exposed cells, a decrease in lactate and glutamate levels, together with an increase in glucose concentration, was found. The AA-induced reduction in glutamate was significantly inhibited by NEB. These findings confirm the involvement of oxidative stress in AA toxicity for endothelial cells and the potential benefit of NEB in preventing endothelial injury.

Keywords: aristolochic acids; nebivolol; endothelial cell; oxidative stress; metabolomic

Key Contribution: Metabolomic analyses highlighted oxidative stress pathways induced in cultured endothelial cells intoxicated by aristolochic acids. Nebivolol pre-treatment demonstrated promising antioxidant properties.

\section{Introduction}

Aristolochic acids (AAs) are active compounds contained in medicinal plants from the Aristolochia and Asarum species. The consumption of AAs is now recognized as a cause of iatrogenic and environmental chronic kidney disease (CKD), namely, aristolochic acid nephropathy (AAN) [1,2]. The onset and progression of renal fibrosis are experimentally characterized by an acute phase (i.e., tubular necrosis of the S3 segment of the proximal tubule reflecting acute kidney injury (AKI)) followed by marked tubular atrophy and interstitial fibrosis leading to end-stage kidney disease [3]. Beside these well-established histologic features of AAN, marked capillary rarefaction has been observed [4,5]. The reduction in vascular density is thought to promote hypoxia and could represent a predisposing factor for the progression of renal failure [6,7]. Peritubular capillary rarefaction has actually been used as a marker to evaluate the long-term impact of AKI-to-CKD progression in experimental models [8]. Among the mechanisms explaining capillary loss, 
the death of endothelial cells by apoptosis via the PI3K/Akt signaling pathway has been proposed [9]. Our studies have previously demonstrated that AAs induce oxidative stress in endothelial cells [10]. Excessive production of reactive oxygen species (ROS) may contribute to decreased nitric oxide (NO) bioavailability, leading to endothelial dysfunction and apoptosis or necrosis [11,12]. Moreover, Yang et al. demonstrated that the protein expression of endothelial nitric oxide synthase (eNOS) was reduced by AAs in a mouse model of AAN [13]. Restoring the bioavailability of NO by reducing oxidative stress has been shown to improve renal function in AA-treated mice [14].

Despite the limited use of AA-containing products, humans might be exposed to AAs accidentally or because of insufficient knowledge about the toxicity of plants containing AAs.

Given the lack of treatments for AAN, nebivolol (NEB), a third-generation beta-blocker drug indicated for the treatment of hypertension, may be of interest. Both in vitro and in vivo studies have shown the antioxidant potential of NEB $[15,16]$. NEB is a highly selective $\beta 1$-blocker with the ability to stimulate eNOS, enhancing NO bioavailability $[17,18]$. Since capillary depletion could represent a predisposing factor for the progression of renal failure, it seems interesting to preserve vascular integrity during toxic oxidative insults.

Metabolomic technology can provide mechanistic insights into the effects of exposure to a toxic compound $[19,20]$. In particular, ${ }^{1} \mathrm{H}-\mathrm{NMR}$-based metabolomics has been reported as a powerful method for studying metabolic responses during rat or human exposure to AAs [21,22]. The aim of the present study was to investigate, in vitro, the effect of NEB in human endothelial cells exposed to AA. A metabolomic approach using ${ }^{1} \mathrm{H}-\mathrm{NMR}$ was also adopted to better characterize the metabolic impact of AA in the presence and absence of NEB.

\section{Results}

\subsection{Nebivolol Protects EAhy926 Cells against AA-Induced Cytotoxicity}

The cytotoxic concentration range for AA in the endothelial cell line was determined in our previous study [10]. Using the EAhy926 cell line, we confirmed that exposure to $50 \mu \mathrm{M}$ AA for $24 \mathrm{~h}$ and $48 \mathrm{~h}$ significantly reduced the viability of EAhy 926 cells compared to a control group (Figure 1A,B). In our experiments, endothelial cells were pretreated with different doses of NEB. The beneficial effect of NEB on AA cell toxicity was more pronounced at $24 \mathrm{~h}$ than $48 \mathrm{~h}$ (Figure 1A,B). Moreover, $10 \mathrm{nM}$ NEB, a concentration close to the clinical plasma concentration [23], attenuated AA-induced cell toxicity more significantly compared to other doses (Figure 1A,B). We found that $10 \mathrm{nM} \mathrm{NEB}$ alone significantly increased cell viability at $24 \mathrm{~h}$ (data not shown). This dose was revealed to be safe as NEB toxicity by itself was detected at $150 \mathrm{nM}$ (data not shown). The cell toxicity induced by AA was illustrated by phase-contrast images, showing a decrease in the number of cells and a change in the morphology of cells, which became elongated upon AA exposure (Figure 1C,D). In experimental conditions, $10 \mathrm{nM}$ NEB restored the number and morphology of cells (Figure 1C,D). 
24h

A

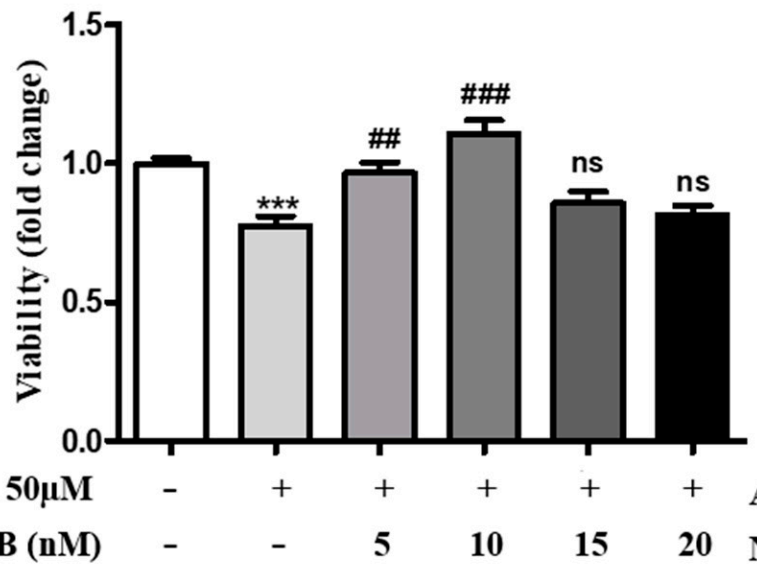

B

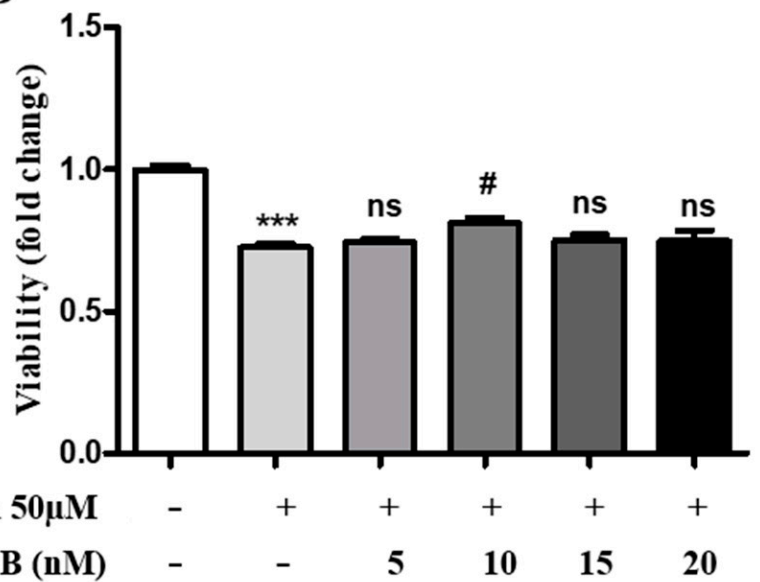

C
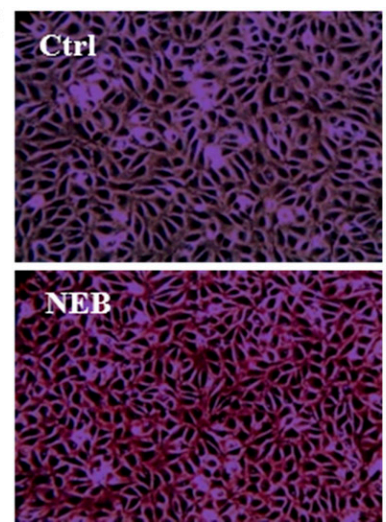
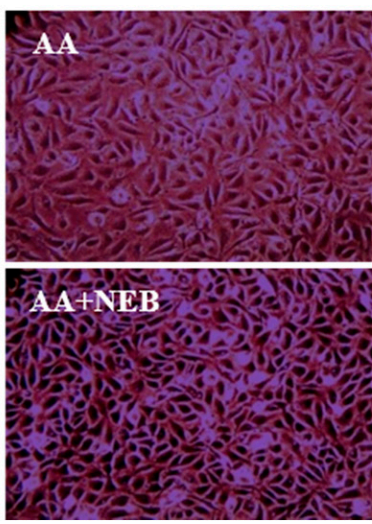

D
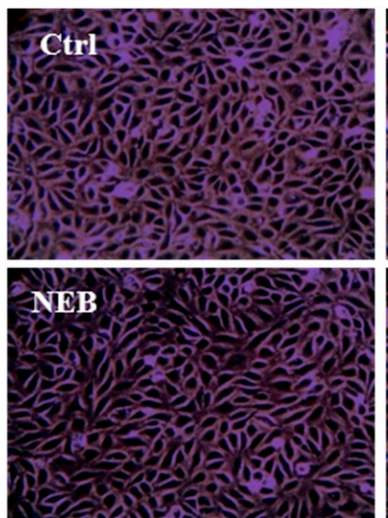
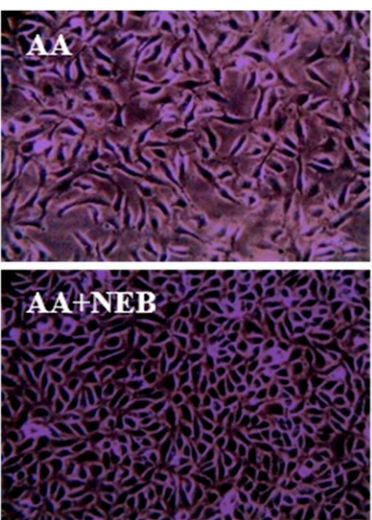

Figure 1. Effect of NEB on AA endothelial cells toxicity (EAhy926). Viability was determined using the resazurin test on cells cultured for $24 \mathrm{~h}(\mathbf{A})$ or $48 \mathrm{~h}$ (B) under standard culture conditions or in the presence of $50 \mu \mathrm{M}$ AA and different concentrations of $\operatorname{NEB}(5,10,15$ and $20 \mathrm{nM})$. Representative images of EAhy926 cells' morphology under standard culture conditions or in the presence of $50 \mu \mathrm{M}$ $\mathrm{AA}$ and/or $10 \mathrm{nM}$ NEB for $24 \mathrm{~h}$ (C) or $48 \mathrm{~h}$ (D) $(200 \times$ magnification). Values are mean \pm SEM showing the fold changes in viability relative to untreated cells. Each assay was completed at least three times, with six biological replicates each. Statistical analysis was performed by one-way ANOVA followed by Bonferroni's multiple comparison test. ns, not significant; ${ }^{* * *} p<0.001$ vs. controls; $\# p<0.05$, \#\# $p<0.01$ and \#\#\# $p<0.001$ vs. AA.

\subsection{Nebivolol Attenuates ROS Levels}

The production of ROS was evaluated by the enhancement of the $2^{\prime}, 7^{\prime}$-dichlorodihydro fluorescein diacetate (DCFDA) fluorescence intensity. As shown in Figure 2, ROS production was significantly increased in cells exposed to AA for $24 \mathrm{~h}$ compared to controls. Importantly, preincubation with NEB significantly inhibited the increased levels of ROS induced by AA, suggesting that NEB can decrease the production of toxic free radicals. 


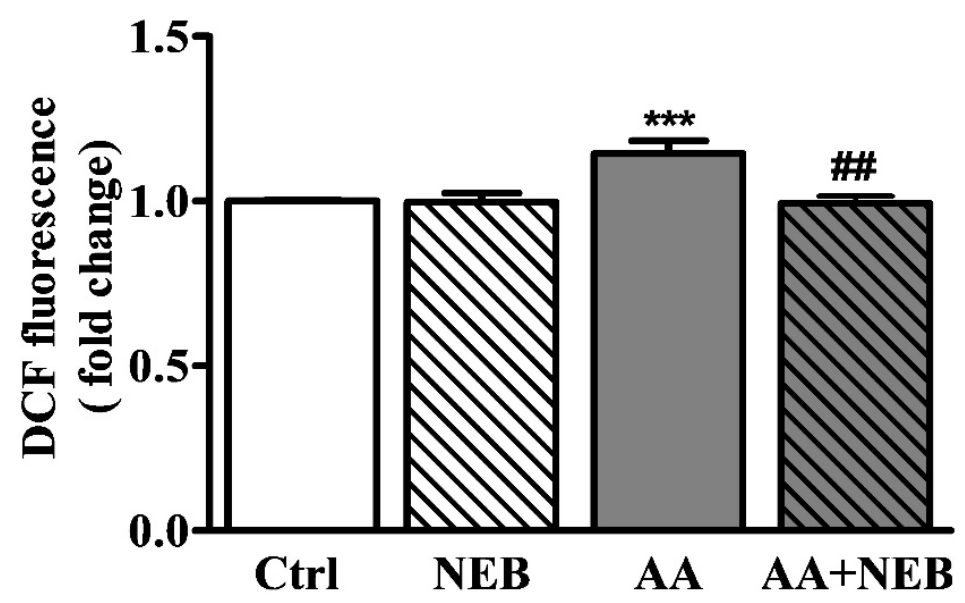

Figure 2. Fluorescent intensity of intracellular ROS analyzed by DCFDA in EAhy926 control cells and in cells exposed to $50 \mu \mathrm{M}$ AA and/or $10 \mathrm{nM}$ NEB for $24 \mathrm{~h}$. Values are mean \pm SEM showing the fold changes in fluorescence relative to untreated cells. Each assay was completed at least six times, with two biological replicates each. Statistical analysis was performed by one-way ANOVA followed by Bonferroni's multiple comparison test. ${ }^{* * *} p<0.001$ vs. controls and \#\# $p<0.01$ vs. AA.

\subsection{Metabolism of EAhy926 Endothelial Cells under Baseline Conditions}

To demonstrate the basal consumption of EAhy926 endothelial cells, the supernatant from endothelial cells was compared with cell-free culture medium. A visual examination of the ${ }^{1} \mathrm{H}-\mathrm{NMR}$ spectra revealed a wide variety of metabolite resonances, which were assigned to specific metabolites according to the public NMR database (Human Metabolome Database (HMDB, Edmonton, AB, Canada,)). In the cell supernatants, a decrease in glucose and amino acids such as glutamine, valine, leucine, isoleucine and arginine, and an increase in lactate compared with the uncultured medium, were observed, reflecting the consumption of glucose and amino acids and the production of lactate by the endothelial cells (Figure 3A).

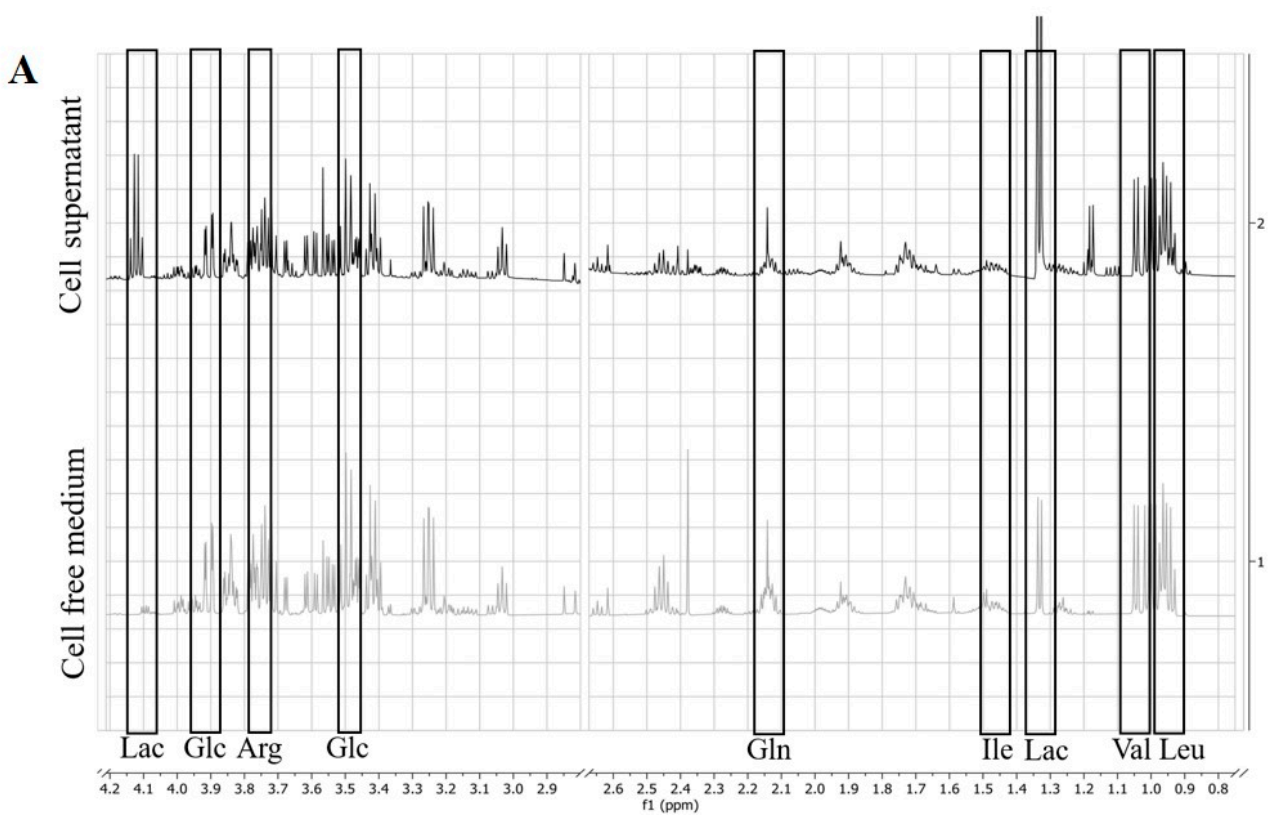

Figure 3. Cont. 
B

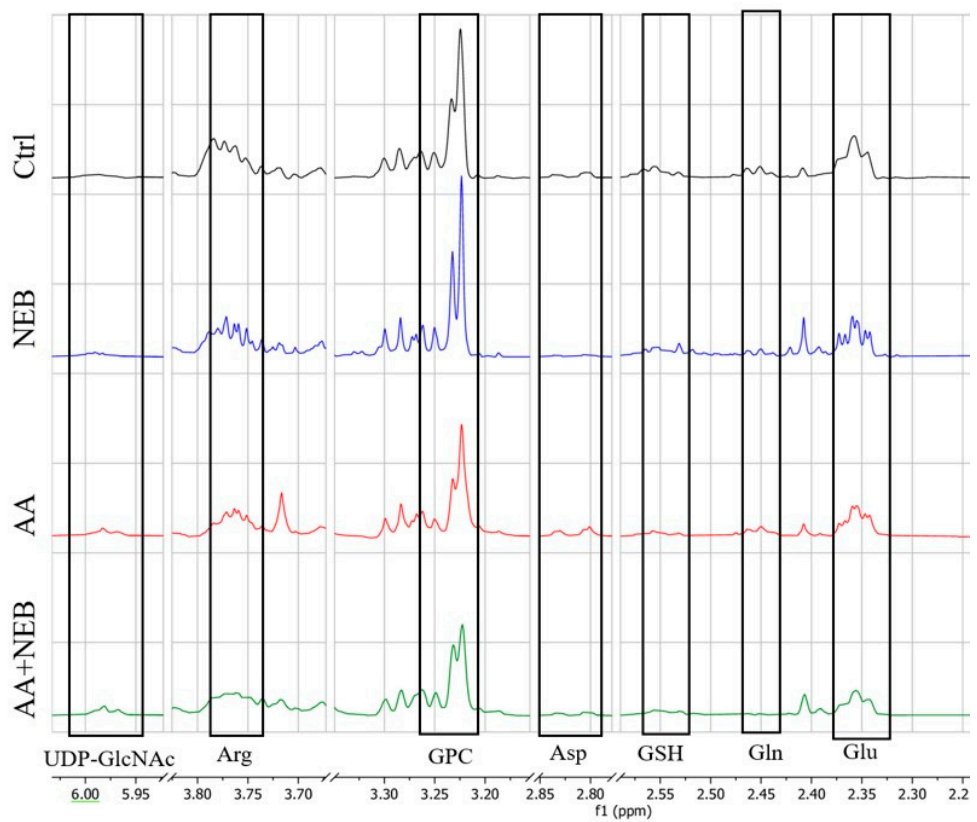

Figure 3. ${ }^{1} \mathrm{H}-\mathrm{NMR}$ spectra $(600 \mathrm{MHz})$ of cell supernatant (black) and cell free medium (grey) (A). ${ }^{1} \mathrm{H}-\mathrm{NMR}$ spectra $(600 \mathrm{MHz})$ of intracellular extract from control group (black), $10 \mathrm{nM}$ NEB group (blue), $50 \mu \mathrm{M}$ AA group (red) and AA + NEB group (green) (B). Arg: arginine; Asp: aspartate; Glc: glucose; Gln: glutamine; Glu: glutamate; GPC: glycerophosphocholine; GSH: glutathione; Ile: isoleucine; Lac: lactate; Leu: leucine; UDP-GlcNAC: UDP-N-acetylglucosamine; Val: valine.

\subsection{Intracellular Metabolic Alterations Induced by AA Exposure}

Afterward, we performed ${ }^{1} \mathrm{H}$-NMR spectroscopy on aqueous-phase endothelial cell extract incubated with and without $50 \mu \mathrm{M}$ AA and/or $10 \mathrm{nM}$ NEB for $48 \mathrm{~h}$ to assess the global metabolic impact of each experimental intervention. Analysis of the NMR spectra of the cell extract highlighted the variety of metabolites identified by HMDB (Figure 3B).

For a comprehensive observation of the metabolic profiles from the four groups and to maximize the distinction between groups, multiple data analyses were performed on the respective NMR data for the cell extract. The score plot obtained from PLS-DA revealed a clear discrimination between the metabolomic profiles obtained from both AA-treated groups (Figure 4A to the left, the AA group (in red) and the AA/NEB group (in green)) and the groups not exposed to AA (to the right, the control group (CTRL in black) and the NEB group (in blue)). A clear separation between the CTRL and NEB groups was also observed. The discrimination between the AA and NEB/AA groups was less obvious. The significant discriminant metabolites were identified from their corresponding descriptors in the loading plots (Figure 4B). Only metabolites characterized by VIP scores higher than one were considered. The corresponding loading plots highlighted metabolites related to oxidative stress such as glutathione, glutamine, glutamate, arginine, glycerophosphocholine, UDP-N-acetylglucosamine and aspartate.

The metabolic changes induced by AA and/or NEB treatment were quantified by calculating the relative spectra's peak area ratios normalized by the total AUC of each spectrum. As shown in Table 1, the most prominent changes induced by AA include significant decreases in arginine, glutamate, glutamine and glutathione as well as significant increases in aspartate, glycerophosphocholine and UDP-N-acetylglucosamine. Pretreating cells with NEB slightly inhibited the changes in glutathione and glycerophosphocholine. 

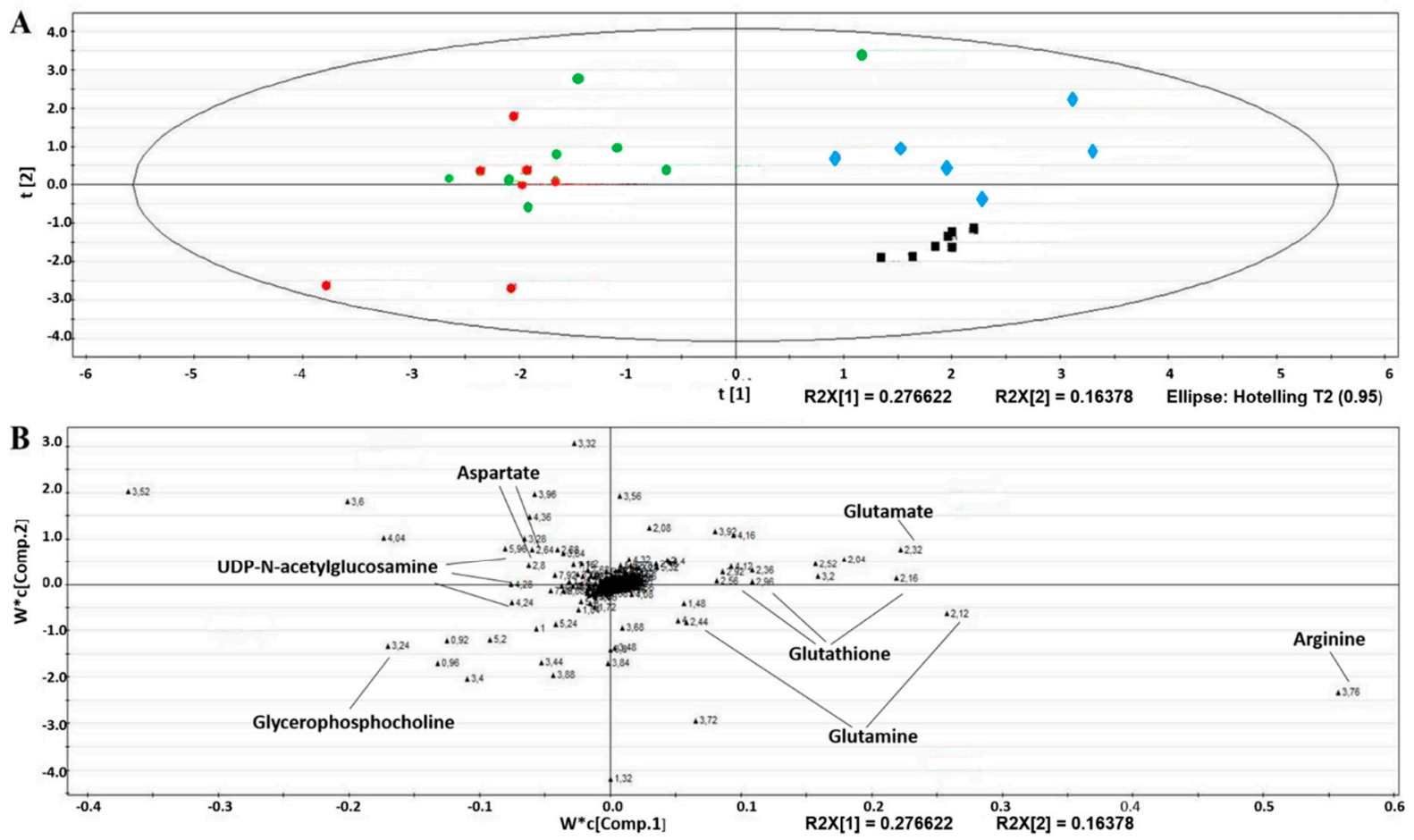

Figure 4. Score plots (A) and corresponding loading plots (B) of the partial least-square (PLS-DA) analysis based on the ${ }^{1} \mathrm{H}-\mathrm{NMR}$ spectra of endothelial cellular extracts from the control (black), $10 \mathrm{nM}$ NEB (blue), $50 \mu \mathrm{M}$ AA (red) and AA + NEB (green) groups.

Table 1. Identified discriminant metabolites with corresponding chemical shifts for $48 \mathrm{~h}$ exposure. Note: Metabolite AUC changes are indicated by arrows. Data are expressed as mean \pm SEM of at least six independent experiments. Statistical analysis was performed by one-way ANOVA followed by Sidak's multiple comparisons test. ${ }^{*} p<0.05,{ }^{* *} p<0.01,{ }^{* * *} p<0.001$ and ${ }^{* * * *} p<0.0001$ vs. control ${ }^{\#} p<0.05$ and ${ }^{\# \#} p<0.01$ vs. AA.

\begin{tabular}{cccc}
\hline Metabolites & $\begin{array}{c}\text { Chemical Shift } \\
(\mathbf{p p m})\end{array}$ & AA vs. Ctrl & AA + NEB vs. AA \\
\hline 1. Intracellular Extracts & & $\downarrow^{* * * *}$ & - \\
\hline Arginine & 3.76 & $\uparrow^{*}$ & - \\
Aspartate & 2.82 & $\downarrow^{*}$ & - \\
Glutamate & 2.32 & $\downarrow^{*}$ & $\uparrow^{* * *}$ \\
Glutamine & 2.44 & $\uparrow^{*}$ & $\downarrow^{*}$ \\
Glutathione & 2.54 & $\uparrow^{* *}$ & - \\
Glycerophosphocholine & 3.24 & $\uparrow^{*}$ & - \\
UDP-N-acetylglucosamine & 5.96 & $\downarrow^{* * *}$ & $\uparrow^{* * *}$ \\
\hline 2. Extracellular Fluids & & - \\
\hline Glucose & 3.4 & & \\
\hline Glutamate & 2.32 & 1.32 &
\end{tabular}

Analysis using MSEA showed that the pathways involved in glutamate, aspartate and glutathione metabolism were significantly associated with the effects of AA. The citrullinearginine cycle and malate-aspartate shuttle were also disrupted by AA (Figure 5A). 

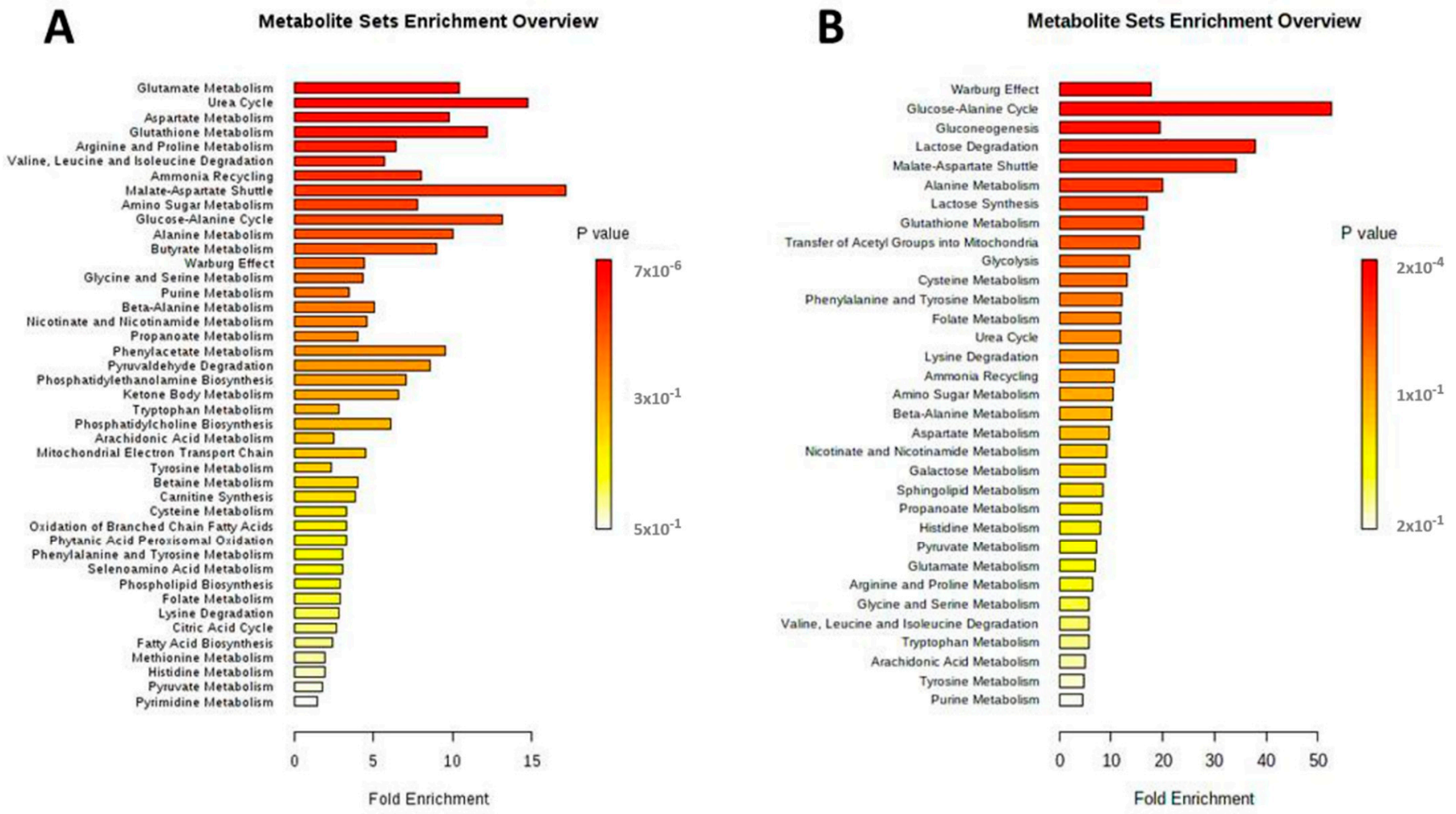

Figure 5. Graphic synopsis of metabolite set enrichment analysis (MSEA) of endothelial cellular extract (A) and extracellular supernatant (B). Results are expressed in a horizontal bar graph showing the most significant metabolite sets identified during analysis. Bars' colors are based on $p$ values (lower $p$ values correspond to darker red), while bars' lengths are based on the fold enrichment.

\subsection{Extracellular Metabolic Alterations Induced by AA Exposure}

The most relevant extracellular metabolites identified by HMDB are labelled in Table 1. In the cell supernatants, a decrease in lactate and glutamate and an increase in glucose were observed in the AA group compared to controls. The AA-induced decrease in glutamate was significantly inhibited by NEB. MSEA revealed that the most significantly altered metabolic pathways associated with the effects of AA were those involving glycolysis, the glucose-alanine cycle, gluconeogenesis, the metabolism of alanine, glutathione and the malate-aspartate shuttle (Figure 5B).

\section{Discussion}

Although AAs, components of many medicinal plants of the Aristolochia and Asarum species, have been widely demonstrated to be nephrotoxic, mainly for the proximal tubular cells, a growing body of evidence illustrates that capillary rarefaction plays an important role in AA-induced AKI $[6,7,24]$. Since few studies have investigated AAs' effects on the endothelial cell, it seemed interesting to evaluate their effects on EAhy926 cells, an endothelial cell line commonly used in studies described in the literature. In the present study, the endothelial cell toxicity of AA was demonstrated by a decreased viability, a change in morphology and the production of ROS. A growing number of studies show that ROS play an important role in AAN $[25,26]$. Moreover, we previously demonstrated that AA produced oxidative stress in endothelial cells, which may contribute to endothelial dysfunction and cell death [10].

Effective therapeutic strategies to prevent or slow down the renal lesions induced by AA are still lacking. Reducing oxidative stress could constitute a valuable approach. Therefore, we examined the effect of NEB, a beta-blocker known for its antioxidant properties. The results of this study show that pretreatment with NEB significantly restored the cell viability and cell morphology and reduced the ROS production induced by AA in 
endothelial cells. Interestingly, NEB alone was found to improve endothelial cell viability as compared to the untreated cells. The underlying mechanism should be investigated. The effect of NEB on cell viability was more marked using $10 \mathrm{nM}$ and after $24 \mathrm{~h}$ of AA exposure, emphasizing the importance of early treatment. The inhibition of ROS production observed in NEB-pretreated cells could be explained, in part, by the antioxidant capacity of NEB, which has been related to its radical-scavenging activity $[27,28]$ and to its capacity to inhibit NADPH oxidase (NOX) activity [17]. Some studies have suggested that one of the first events leading to the onset and progression of oxidative stress in the vascular system was a decrease in the bioavailability of NO [15]. A growing number of studies have shown that NEB is able to increase eNOS activity following the stimulation of $\beta 3$-adrenergic receptors $[16,18]$. According to other authors, NEB is able to reduce the endogenous concentration of asymmetric dimethylarginine (ADMA), an eNOS inhibitor [29]. This increase in eNOS activity induced by NEB could contribute to restoring the bioavailability of NO. Indeed, AA-induced oxidative stress is one mechanism of endothelial dysfunction, as superoxide rapidly inactivates $\mathrm{NO}$ and forms peroxynitrite. Consequently, through its antioxidant action and the restoration of NO, NEB could attenuate endothelial dysfunction.

To better understand the mechanism of AA cytotoxicity and the beneficial effect of NEB on endothelial cells, a metabolomic approach was adopted. ${ }^{1} \mathrm{H}-\mathrm{NMR}$ has been demonstrated to be a valuable approach for studying the oxidative stress response to chemical perturbations [30]. Both intra- and extracellular compartments of EAhy926 cells were analyzed. The baseline experimental conditions (without any toxic agent) of the endothelial cells were first analyzed by examining the extracellular medium. As expected, we observed a consumption of glucose, glutamine and amino acids such as valine, leucine and isoleucine, which represent major substrates for the energy production of the endothelial cell [31,32]. The lactate production observed in our experiments is in agreement with the data of De Bock et al. These authors observed, in human umbilical vein endothelial cells (HUVECs), that the glycolytic pathway was 200 times more active than the glucose oxidation pathway [33], which is consistent with the mitochondrial volume being relatively low in the endothelial cells [34].

After $48 \mathrm{~h}$ of cell exposure to AA, analysis of the aqueous intracellular extracts highlighted major metabolic alterations. The most prominent changes included a significant decrease in glutathione, glutamate, glutamine and arginine. The metabolite set enrichment analysis (MSEA) of the endothelial cellular extracts revealed metabolic pathways related to oxidative stress such as glutathione and glutamate metabolism. Glutathione is involved in defending cells against both physiologically and pathologically generated oxidative stress. It is also involved in detoxification processes. Zhang et al. demonstrated that the aristolactam-nitrenium ion intermediate also reacted with glutathione in vitro and in rats, producing phase-two conjugated metabolites [35]. The decrease in glutathione and its precursors glutamate and glutamine in the intracellular compartment could reflect consumption intended either to fight against the oxidative stress induced by AA or to detoxify AA's metabolites by conjugation reactions.

The NEB pretreatment of AA-exposed cells significantly, but still only partially, restored the glutathione levels, meaning that NEB exerts its antioxidant effects through other mechanisms. In our study, arginine, the substrate for NO, was found to be significantly decreased in cells exposed to AA compared with in the control cells. MSEA of the endothelial cellular extract revealed that aspects of the urea cycle corresponding to the citrullinearginine cycle in the endothelial cells and arginine/proline metabolism were perturbed. The involvement of the citrulline-arginine cycle in the restoration of arginine levels in endothelial cells was described by Hecker et al. and facilitates optimal NO signaling [36,37]. Our experiments showed that NEB was able to inhibit the decrease in arginine due to AA exposure, leading to enhanced eNOS activity and contributing, therefore, to restoring NO bioavailability. Moreover, Mihout et al. suggested that ADMA, known to inhibit eNOS by competing with arginine, contributes to endothelial dysfunction during the development 
of renal fibrosis [38]. The ability of NEB to reduce the endogenous concentration of ADMA could explain the restoration of NO bioavailability [29].

We also observed, in our experiments, an increase in aspartate. Aspartate is generally catabolized into intermediates that enter the TCA cycle, reflecting its lower consumption as a glucogenic amino acid during AA exposure. This observation clearly indicates an alteration or dysfunction of the mitochondria induced by AA. Similar findings supporting the hypothesis of Krebs cycle disruption by AA have been reported in other studies [21,39]. MSEA demonstrated dysfunction of the malate-aspartate shuttle. The malate-aspartate shuttle translocates reducing equivalents from the cytoplasm to mitochondria. The changes in the levels of glutamate observed in our study could inhibit the malate-aspartate shuttle and oxidative phosphorylation [40]. An increase in glucosamine levels, and especially UDP-N-acetyl-glucosamine, was also observed. UDP-N-acetyl-glucosamine is the endproduct of the hexosamine metabolic pathway produced from glucose derivatives. This metabolic pathway is closely related to glycolysis. UDP-N-acetyl-glucosamine is involved in glycosylation processes. The alteration of this mechanism can lead to an accumulation of malformed proteins, endoplasmic reticulum stress, and, in severe cases, an apoptotic response. In our experiments, it seemed that the hexosamine pathway was abnormally activated by AA, as the levels of UDP-N-acetyl-glucosamine increased while those of glutamine decreased. Some authors have observed that glycosylation protects proteins against free radicals generated from toxic xenobiotics [41]. From this, it would be tempting to argue that the activation of glycosylation is part of a defense mechanism protecting against the free radicals induced by AA. Our approach also revealed a significant increase in glycerophosphocholine. This result is consistent with previous studies that showed an increase in glycerophosphocholine in the renal tissue of AA-treated rats [42,43]. Glycerophosphocholine plays an important role in the structural integrity of the cell membrane [44]. Some authors suggest that the increase in glycerophosphocholine could be a mechanism protecting against the cell damage induced by oxidative stress $[45,46]$. Furthermore, in the presence of NEB, known for its antioxidant properties, a lower increase in glycerophosphocholine was observed.

The metabolomic analysis of extracellular media can provide information on the metabolites released by the cells and on the substances contained in the culture medium that have not been consumed by the cells. In our experiments, the analysis of cell culture supernatants showed a decrease in lactate and glutamate as well as an increase in glucose in the extracellular medium of cells exposed to AA, indicating that both glycolytic metabolism and mitochondrial glucose oxidation were downregulated during AA exposure. Several authors have shown mitochondrial dysfunction in AA-related nephrotoxicity $[26,47]$. The subsequent alteration of the Krebs cycle could explain the decrease in glucose consumption and, hence, greater concentration of this metabolite in the extracellular medium [48]. In addition, a slowdown in lactate production should not be ruled out given the decreased lactate content in the extracellular environment of the cells exposed to AA. The increased activity of the hexosamine biosynthetic pathway observed in the AA-exposed cells could participate in the reduction of lactate excretion. The culture medium contains glutamine and is devoid of glutamate. Therefore, it is most likely that the glutamate observed in the medium comes from an efflux of glutamate through a transporter. The cystine/glutamate transporter allows the uptake of cystine via exchange with glutamate across the cell membrane for glutathione (GSH) biosynthesis to alleviate oxidative stress [49]. In our experiments, a decreased glutamate level was observed in the extracellular medium from cells incubated with AA. High glutamate export in the oxidative condition could result in a partial depletion of intracellular glutamate, leading to a decreased efflux of glutamate [50]. The decreased glutamate observed in the intracellular compartment, probably due to increased synthesis of glutathione, either to fight against oxidative stress or to detoxify the metabolites of AA by conjugation reactions, could also explain the reduced glutamate efflux. 


\section{Conclusions}

We provide evidence that the exposure of endothelial cells to AA results in cytotoxicity associated with oxidative stress and metabolic alterations that could lead to cell death. Our metabolomic analyses highlight AA-induced changes in metabolites involved in metabolic pathways such as the glutamate, glutathione aspartate, arginine metabolism, citrulline-arginine and Krebs cycles. As shown in Figure 6, all these results provide a preliminary demonstration of AA-induced alterations, consistent not only with oxidative stress and mitochondrial dysfunction leading to endothelial damage but also with defense mechanisms protecting against free radicals. The reduction of oxidative stress could be one of the ways to mitigate the toxic effects of AA in endothelial cells and the subsequent disappearance of microvasculature. The antioxidant properties of NEB could partly explain its cytoprotective effects on endothelial cells damaged by AA.

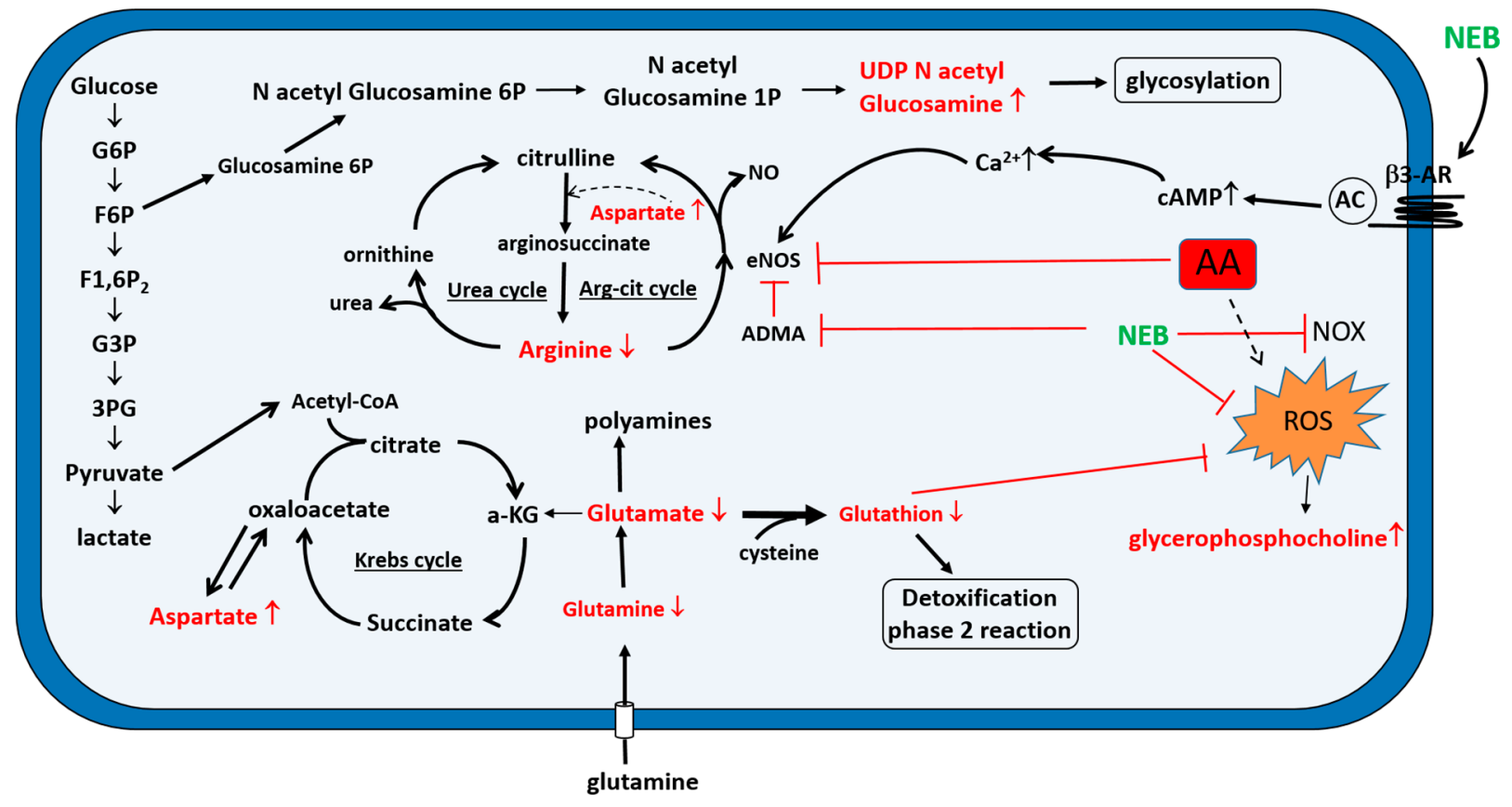

Figure 6. Proposed metabolic pathway affected by AA and the protective effects of NEB. AAs increase the production of ROS, which leads the cell to fight oxidative stress (1) through glutathione and its precursors (glutamate and glutamine) and (2) by promoting glycosylation that protects proteins against free radicals. AAs decrease the expression of eNOS and the bioavailability of NO. AAs affect the Krebs cycle and citrulline-arginine (cit-arg) cycle, with decreased arginine production and increased aspartate. NEB decreases ROS (1) by the scavenger effect and (2) by activating eNOS following its binding to the $\beta 3$ adrenergic receptor (AR) and inhibiting ADMA, which leads to an increase in the bioavailability of NO. $\uparrow=$ increase; $\downarrow=$ decrease; $\perp=$ inhibition; $\rightarrow=$ activation.

\section{Materials and Methods}

\subsection{Chemicals}

A mixture of AAI and II (50\% of each compound) was purchased from Acros Organics Co. (Geel, Belgium). Dulbecco's Modified Eagle Medium (DMEM), penicillin/streptomycin, L-glutamine and accutase were obtained from Capricorn Scientific (Ebsdorfergrund, Germany). Fetal bovine serum (FBS) was purchased from Life Technologies Europe (Gent, Belgium), and sodium citrate, from Merck (Darmstadt, Germany). Resazurin, $2^{\prime}$, $7^{\prime}$-dichlorodihy drofluorescein diacetate (DCFDA) and nebivolol hydrochloride were obtained from Sigma (St. Louis, MO, USA). 


\subsection{Cell Cultures}

The human endothelial cell line EAhy926 was purchased from the American Type Culture Collection (ATCC, Molsheim, France), and the cells were grown in a DMEM highglucose medium supplemented with $10 \% \mathrm{FBS}$ and $1 \%$ penicillin/streptomycin at $37^{\circ} \mathrm{C}$ under $5 \% \mathrm{CO}_{2}$. When they had reached about $80-90 \%$ confluence, the cells were harvested and seeded on 96 -well plates $\left(1 \times 10^{4}\right.$ cells), six-well plates $\left(4 \times 10^{5}\right.$ cells $)$ and a $75 \mathrm{~cm}^{2}$ flask $\left(5 \times 10^{6}\right.$ cells) from Greiner bio-One (Vilvoorde, Belgium). The cells were then incubated in complete medium for $24 \mathrm{~h}$ and treated with $50 \mu \mathrm{M}$ AA and/or different doses of NEB (5, $10,15,20 \mathrm{nM}$ ) in FBS-depleted low-glucose DMEM for $24 \mathrm{~h}$ or $48 \mathrm{~h}$.

\subsection{Cell Viability}

The cytotoxicity of the tested samples was assessed by the resazurin reduction assay. This assay is based on the reduction of the indicator dye, resazurin, to the highly fluorescent resorufin by viable cells. Cells were treated with $50 \mu \mathrm{M}$ AA and different doses of NEB (5, $10,15,20 \mathrm{nM}$ ) in 96-well plates. After 24 or $48 \mathrm{~h}$, the cells were washed twice with PBS and incubated with $0.44 \mathrm{mM}$ resazurin solution at $37^{\circ} \mathrm{C}$ for $2 \mathrm{~h}$. The absorbance at 540 and $620 \mathrm{~nm}$ wavelengths was measured using an iEMS Reader MF spectrophotometer (Thermo Labsystems, Breda, The Netherlands). Each assay was performed at least three times, with six replicates each.

\subsection{Microscopic Examination}

To evaluate the impact of AA on the morphology, EAhy926 cells were incubated with $50 \mu \mathrm{M} \mathrm{AA}$ and/or $10 \mathrm{nM}$ NEB for $24 \mathrm{~h}$ and $48 \mathrm{~h}$ in six-well plates. The cells were imaged using a Motic AE21 phase-contrast microscope equipped with a Moticam 2300 camera (Motic, Wetzlar, Germany) at a magnification of $200 \times$.

\subsection{Measurement of ROS}

Following $24 \mathrm{~h}$ of treatment in six-well plates, cells were equilibrated for $10 \mathrm{~min}$ in HBSS at $37^{\circ} \mathrm{C}$ and treated with $5 \mu \mathrm{M}$ DCFDA solution at $37^{\circ} \mathrm{C}$ for $15 \mathrm{~min}$. Afterward, the cells were harvested with accutase, centrifuged and analyzed by flow cytometry (FACS Canto II, BD Biosciences, San Jose, CA, USA) for ROS detection. The DCFDA fluorescence was measured at an excitation wavelength of $488 \mathrm{~nm}$ and an emission wavelength of $535 \mathrm{~nm}$ (FL-1). A total of $10^{4}$ cells were recorded, and the mean fluorescence intensities were estimated and are expressed as percentages of the untreated control.

\section{6. ${ }^{1} \mathrm{H}-\mathrm{NMR}$ Samples' Collection and Extraction}

The $48 \mathrm{~h}$ culture media were transferred from $75 \mathrm{~cm}^{2}$ tissue culture flasks into sterile $15 \mathrm{~mL}$ conical tubes and centrifuged at $1500 \times \mathrm{g}$ for $5 \mathrm{~min}$ at room temperature to pellet any cellular debris. The remaining cells were rinsed twice with $7 \mathrm{~mL}$ of ice-cold phosphatebuffered saline ( $\mathrm{pH}$ 7.4). The cells were quenched using $3 \mathrm{~mL}$ of ice-cold methanol to terminate metabolic reactions and then harvested by scraping. The cell suspension was then mixed with methanol:water:chloroform (1:0.9:1) to separate the aqueous phase from the organic phase. The supernatant was transferred to fresh tubes, dried and stored at $-80{ }^{\circ} \mathrm{C}$ until further evaluation. The polar phase containing the intracellular metabolite mixture was dried using a SpeedVacuum and stored at $-80^{\circ} \mathrm{C}$ before analysis.

\section{7. ${ }^{1} H-N M R$ Spectroscopy}

The dried residues were reconstituted with $700 \mu \mathrm{L}$ of phosphate buffer prepared in a mixture of $\mathrm{H}_{2} \mathrm{O} / \mathrm{D}_{2} \mathrm{O}(80: 20 ; v: v)$. Following vortexing and centrifugation at $13,000 \times g$ for $10 \mathrm{~min}, 650 \mu \mathrm{L}$ of supernatant was mixed with $50 \mu \mathrm{L}$ of a $7 \mathrm{mM}$ solution of 3-trimethylsilyl propionic-2,2,3,3-d4 acid (TSP) prepared in 100\% deuterium oxide and transferred into $5 \mathrm{~mm}$ NMR tubes for NMR analyses. The acquisition of the ${ }^{1} \mathrm{H}-\mathrm{NMR}$ spectra was performed on a Bruker 600-MHz NMR spectrometer (Bruker Avance, Germany) using the 
NOESYPRESAT-1D pulse sequence, 128 scans, 32,768 data points, a spectral width of $6410.2 \mathrm{~Hz}$, an acquisition time of $2.5 \mathrm{~s}$ and a pulse recycle delay of $2 \mathrm{~s}$.

\subsection{Multivariate Data Analyses and Metabolite Identification}

The MestRe Nova 10 software (Mestre Lab Research, Santiago de Compostela, Spain) was used for the phase and baseline corrections of all the spectra. The chemical shift was referenced to TSP and binned in subregions of $0.04 \mathrm{ppm}$ sections. The spectral binned data were converted to the Microsoft Excel format, and the AUC of one isolated chemical shift, previously normalized using the total AUC of each spectrum, was calculated. The data were imported into the SIMCA-P+ 12.0 software (Umetrics, Umeå, Sweden) for multivariate statistical analysis. Principal component analysis (PCA) followed by a partial least-square discriminant analysis (PLS-DA) was performed to examine the intrinsic variation in the dataset. PCA was used to highlight the possible group separation observed in the score plot and to identify potential outliers. PLS-DA was used to identify significant discriminant descriptors (metabolites). These metabolites were ranked according to their variable influence on projection (VIP) scores. When VIP $>1$, the variables were considered statistically significant variables and their corresponding metabolites were identified. Metabolites were identified using the Chenomx NMR suite software (version 8.1.1) (Edmonton, Canada) and the human metabolome database (HMDB). To highlight the biological pathways possibly linked to the discriminant metabolites, the MetaboAnalist 4.0 online software for metabolite set enrichment analysis (MSEA) was used.

\subsection{Statistical Analyses}

All the data for cell viability and oxidative stress are expressed as the means $\pm \mathrm{SEMs}$. Differences between cell culture conditions were evaluated by analysis of variance (ANOVA) followed by a post hoc analysis (Bonferroni's multiple-comparison test) using Prism 5 (GraphPad Software, Inc., San Diego, CA, USA). $p<0.05$ was considered significant. Regarding the metabolomics, statistical significance was determined by integrating the NMR peaks of each of the metabolites that contributed to the multivariate separation using an ANOVA followed by Sidak' multiple comparisons test, and $p<0.05$ was considered significant.

Author Contributions: Conceptualization, M.-H.A., J.-M.C. and J.N.; Data curation, C.H., T.Y. and V.T.; Formal analysis, C.H., T.Y. and V.T.; Funding acquisition, J.-M.C. and J.N.; Investigation, M.-H.A., C.H., T.Y. and S.M.; Methodology, M.-H.A., C.H. and V.T.; Software, C.H., T.Y. and V.T.; Supervision, M.-H.A., V.T. and J.N.; Writing—original draft, M.-H.A. and J.N.; Writing—review and editing, V.T. and J.-M.C. All authors have read and agreed to the published version of the manuscript.

Funding: This work was partially supported by a grant from the "Association de Défense des Insuffisants Rénaux" (ADIR, Brussels, Belgium). The bioprofiling platform including the ${ }^{1} \mathrm{H}-\mathrm{NMR}$ $600 \mathrm{MHz}$ Bruker spectrometer was supported by the European Regional Development Fund and the Walloon Region, Belgium.

Institutional Review Board Statement: Not applicable.

Informed Consent Statement: Not applicable.

Data Availability Statement: Not applicable.

Conflicts of Interest: The authors declare no conflict of interest.

\section{Abbreviations}

AAs: Aristolochic acids; AAN: aristolochic acid nephropathy; ADMA: asymmetric dimethylarginine; AKI: acute kidney injury; AR: adrenergic receptor; Arg: arginine; Asp: aspartate; CKD: chronic kidney disease; DCFDA: $2^{\prime}, 7^{\prime}$-dichlorodihydrofluorescein diacetate; eNOS: endothelial nitric oxide synthase; Glc: glucose; Gln: glutamine; Glu: glutamate; GPC: glycerophosphocholine; GSH: glutathione; HMDB: Human Metabolome Database; ${ }^{1} \mathrm{H}$ NMR: proton nuclear magnetic resonance; HUVECs: human umbilical vein endothelial cells; Ile: isoleucine; Lac: lactate; Leu: leucine; MSEA: metabolite set enrichment analysis; NEB: nebivolol; nm: nanometer; NO: nitric oxide; PCA: 
principal component analysis; PLS-DA: partial least squares discriminant analysis; ROS: reactive oxygen species; TCA cycle: tricarboxylic acid cycle, also called Krebs cycle; UDP-GlcNAC: UDP-Nacetylglucosamine; Val: valine; VIP: variable influence on projection.

\section{References}

1. Vanherweghem, J.L.; Depierreux, M.; Tielemans, C.; Abramowicz, D.; Dratwa, M.; Jadoul, M.; Richard, C.; Vandervelde, D.; Verbeelen, D.; Vanhaelen-Fastre, R.; et al. Rapidly progressive interstitial renal fibrosis in young women: Association with slimming regimen including Chinese herbs. Lancet 1993, 13, 387-391. [CrossRef]

2. Bunel, V.; Souard, F.; Antoine, M.H.; Stevigny, C.; Nortier, J.L. Nephrotoxicity of Natural Products: Aristolochic Acid and Fungal Toxins. In Comprehensive Toxicology, 3rd ed.; McQueen, C.A., Ed.; Elsevier Ltd.: Oxford, UK, 2018; Volume 14, pp. $340-379$.

3. Debelle, F.D.; Nortier, J.L.; De Prez, E.G.; Garbar, C.H.; Vienne, A.R.; Salmon, I.J.; Deschodt-Lanckman, M.M.; Vanherweghem, J.L. Aristolochic acids induce chronic renal failure with interstitial fibrosis in salt-depleted rats. J. Am. Soc. Nephrol. 2002, 13, 431-436. [CrossRef] [PubMed]

4. Sun, D.; Feng, J.; Dai, C.; Sun, L.; Jin, T.; Ma, J.; Wang, L. Role of peritubular capillary loss and hypoxia in progressive tubulointerstitial fibrosis in a rat model of aristolochic acid nephropathy. Am. J. Nephrol. 2006, 26, 363-371. [CrossRef]

5. Depierreux, M.; Van Damme, B.; Vanden Houte, K.; Vanherweghem, J.L. Pathologic aspects of a newly described nephropathy related to the prolonged use of Chinese herbs. Am. J. Kidney Dis. 1994, 24, 172-180. [CrossRef]

6. Menshikh, A.; Scarfe, L.; Delgado, R.; Finney, C.; Zhu, Y.; Yang, H.; de Caestecker, M.P. Capillary rarefaction is more closely associated with CKD progression after cisplatin, rhabdomyolysis, and ischemia-reperfusion-induced AKI than renal fibrosis. Am. J. Physiol. Renal Physiol. 2019, 317, F1383-F1397. [CrossRef] [PubMed]

7. Fine, L.G.; Norman, J.T. Chronic hypoxia as a mechanism of progression of chronic kidney diseases: From hypothesis to novel therapeutics. Kidney Int. 2008, 74, 867-872. [CrossRef]

8. Basile, D.P. Rarefaction of peritubular capillaries following ischemic acute renal failure: A potential factor predisposing to progressive nephropathy. Curr. Opin. Nephrol. Hypertens. 2004, 13, 1-7. [CrossRef]

9. Shi, H.; Feng, J.M. Aristolochic acid induces apoptosis of human umbilical vein endothelial cells in vitro by suppressing PI3K/Akt signaling pathway. Acta Pharmacol. Sin. 2011, 32, 1025-1030. [CrossRef]

10. Youl, E.N.H.; Husson, C.; El Khattabi, C.; El Mere, S.; Declèves, A.E.; Pochet, S.; Nortier, J.L.; Antoine, M.H. Characterization of cytotoxic effects of aristolochic acids on the vascular endothelium. Toxicol. In Vitro 2020, 65, 104811. [CrossRef]

11. Bertocchi, C.; Schmid, M.; Hasslacher, J.; Dunzendorfer, S.; Patsch, J.R.; Joannidis, M. Differential effects of NO inhibition in renal epithelial and endothelial cells in mono-culture vs. co-culture conditions. Cell Physiol. Biochem. 2010, 26, 669-678. [CrossRef]

12. Dubois-Deruy, E.; Peugnet, V.; Turkieh, A.; Pinet, F. Oxidative Stress in Cardiovascular Diseases. Antioxidants 2020, 9, 864. [CrossRef] [PubMed]

13. Yang, X.; Thorngren, D.; Chen, Q.; Wang, M.; Xie, X. Protective role of relaxin in a mouse model of aristolochic acid nephropathy. Biomed. Pharmacother. 2019, 115, 108917. [CrossRef] [PubMed]

14. Declèves, A.É.; Jadot, I.; Colombaro, V.; Martin, B.; Voisin, V.; Nortier, J.; Caron, N. Protective effect of nitric oxide in aristolochic acid-induced toxic acute kidney injury: An old friend with new assets. Exp. Physiol. 2016, 101, 193-206. [CrossRef] [PubMed]

15. Coats, A.; Jain, S. Protective effects of nebivolol from oxidative stress to prevent hypertension-related target organ damage. J. Hum. Hypertens. 2017, 31, 376-381. [CrossRef] [PubMed]

16. Evangelista, S.; Garbin, U.; Pasini, A.F.; Stranieri, C.; Boccioletti, V.; Cominacini, L. Effect of DL-nebivolol, its enantiomers and metabolites on the intracellular production of superoxide and nitric oxide in human endothelial cells. Pharmacol. Res. 2007, 55, 303-309. [CrossRef]

17. Vanhoutte, P.M.; Gao, Y. Beta blockers, nitric oxide, and cardiovascular disease. Curr. Opin. Pharmacol. 2013, 13, 265-273. [CrossRef]

18. Ladage, D.; Brixius, K.; Hoyer, H.; Steingen, C.; Wesseling, A.; Malan, D.; Bloch, W.; Schwinger, R.H. Mechanisms underlying nebivolol-induced endothelial nitric oxide synthase activation in human umbilical vein endothelial cells. Clin. Exp. Pharmacol. Physiol. 2006, 33, 720-724. [CrossRef]

19. Markley, J.L.; Brüschweiler, R.; Edison, A.S.; Eghbalnia, H.R.; Powers, R.; Raftery, D.; Wishart, D.S. The future of NMR-based metabolomics. Curr. Opin. Biotechnol. 2017, 43, 34-40. [CrossRef]

20. Colet, J.M. Metabonomics in the preclinical and environmental toxicity field. Drug Discov. Today Technol. 2015, 13, 3-10. [CrossRef]

21. Duquesne, M.; Declèves, A.E.; De Prez, E.; Nortier, J.L.; Colet, J.M. Interest of metabonomic approach in environmental nephrotoxicants: Application to aristolochic acid exposure. Food Chem. Toxicol. 2017, 108, 19-29. [CrossRef]

22. Mantle, P.; Modalca, M.; Nicholls, A.; Tatu, C.; Tatu, D.; Toncheva, D. Comparative (1)H NMR metabolomic urinalysis of people diagnosed with Balkan endemic nephropathy, and healthy subjects, in Romania and Bulgaria: A pilot study. Toxins 2011, 3, 815-833. [CrossRef] [PubMed]

23. Briciu, C.; Neag, M.; Muntean, D.; Bocsan, C.; Buzoianu, A.; Antonescu, O.; Gheldiu, A.M.; Achim, M.; Popa, A.; Vlase, L. Phenotypic differences in nebivolol metabolism and bioavailability in healthy volunteers. Clujul Med. 2015, 88, 208-213. [CrossRef] [PubMed] 
24. Pozdzik, A.A.; Giordano, L.; Li, G.; Antoine, M.H.; Quellard, N.; Godet, J.; De Prez, E.; Husson, C.; Declèves, A.E.; Arlt, V.M.; et al. Blocking TGF- $\beta$ Signaling Pathway Preserves Mitochondrial Proteostasis and Reduces Early Activation of PDGFR $\beta+$ Pericytes in Aristolochic Acid Induced Acute Kidney Injury in Wistar Male Rats. PLoS ONE 2016, 11, e0157288. [CrossRef] [PubMed]

25. Zhu, S.; Wang, Y.; Jin, J.; Guan, C.; Li, M.; Xi, C.; Ouyang, Z.; Chen, M.; Qiu, Y.; Huang, M.; et al. Endoplasmic reticulum stress mediates aristolochic acid I-induced apoptosis in human renal proximal tubular epithelial cells. Toxicol. In Vitro 2012, 26, 663-671. [CrossRef]

26. Liu, X.; Wang, J.; Wang, J.; Feng, X.; Wu, H.; Huang, R.; Fan, J.; Yu, X.; Yang, X. Mitochondrial dysfunction is involved in aristolochic acid I-induced apoptosis in renal proximal tubular epithelial cells. Hum. Exp. Toxicol. 2020, 39, 673-682. [CrossRef]

27. Mason, R.P.; Kubant, R.; Jacob, R.F.; Walter, M.F.; Boychuk, B.; Malinski, T. Effect of nebivolol on endothelial nitric oxide and peroxynitrite release in hypertensive animals: Role of antioxidant activity. J. Cardiovasc. Pharmacol. 2006, 48, 862-869. [CrossRef]

28. De Groot, A.A.; Mathy, M.J.; van Zwieten, P.A.; Peters, S.L. Antioxidant activity of nebivolol in the rat aorta. J. Cardiovasc. Pharmacol. 2004, 43, 148-153. [CrossRef]

29. Wang, Y.; An, W.; Zhang, F.; Niu, M.; Liu, Y.; Shi, R. Nebivolol ameliorated kidney damage in Zucker diabetic fatty rats by regulation of oxidative stress/NO pathway: Comparison with captopril. Clin. Exp. Pharmacol. Physiol. 2018, 45, 1135-1148. [CrossRef]

30. Dallons, M.; Schepkens, C.; Dupuis, A.; Tagliatti, V.; Colet, J.M. New Insights About Doxorubicin-Induced Toxicity to Cardiomyoblast-Derived H9C2 Cells and Dexrazoxane Cytoprotective Effect: Contribution of In Vitro (1)H-NMR Metabonomics. Front Pharmacol. 2020, 11, 79. [CrossRef]

31. Eelen, G.; de Zeeuw, P.; Treps, L.; Harjes, U.; Wong, B.W.; Carmeliet, P. Endothelial Cell Metabolism. Physiol. Rev. 2018, 98 , 3-58. [CrossRef]

32. Pink, M.; Verma, N.; Rettenmeier, A.W.; Schmitz-Spanke, S. Integrated proteomic and metabolomic analysis to assess the effects of pure and benzo[a]pyrene-loaded carbon black particles on energy metabolism and motility in the human endothelial cell line EA.hy926. Arch. Toxicol. 2014, 88, 913-934. [CrossRef] [PubMed]

33. De Bock, K.; Georgiadou, M.; Schoors, S.; Kuchnio, A.; Wong, B.W.; Cantelmo, A.R.; Quaegebeur, A.; Ghesquière, B.; Cauwenberghs, S.; Eelen, G.; et al. Role of PFKFB3-driven glycolysis in vessel sprouting. Cell 2013, 154, 651-663. [CrossRef] [PubMed]

34. Groschner, L.N.; Waldeck-Weiermair, M.; Malli, R.; Graier, W.F. Endothelial mitochondria-Less respiration, more integration. Pflugers Arch. 2012, 464, 63-76. [CrossRef] [PubMed]

35. Zhang, J.; Chan, C.K.; Ham, Y.H.; Chan, W. Identifying Cysteine, N-Acetylcysteine, and Glutathione Conjugates as Novel Metabolites of Aristolochic Acid I: Emergence of a New Detoxification Pathway. Chem. Res. Toxicol. 2020, 33, 1374-1381. [CrossRef]

36. Wu, X.; Sun, X.; Sharma, S.; Lu, Q.; Yegambaram, M.; Hou, Y.; Wang, T.; Fineman, J.R.; Black, S.M. Arginine recycling in endothelial cells is regulated BY HSP90 and the ubiquitin proteasome system. Nitric Oxide 2021, 1, 12-19. [CrossRef]

37. Hecker, M.; Sessa, W.C.; Harris, H.J.; Anggård, E.E.; Vane, J.R. The metabolism of L-arginine and its significance for the biosynthesis of endothelium-derived relaxing factor: Cultured endothelial cells recycle L-citrulline to L-arginine. Proc. Natl. Acad. Sci. USA 1990, 87, 8612-8616. [CrossRef]

38. Mihout, F.; Shweke, N.; Bigé, N.; Jouanneau, C.; Dussaule, J.C.; Ronco, P.; Chatziantoniou, C.; Boffa, J.J. Asymmetric dimethylarginine (ADMA) induces chronic kidney disease through a mechanism involving collagen and TGF- $\beta 1$ synthesis. J. Pathol. 2011, 223, 37-45. [CrossRef]

39. Cui, Y.; Han, J.; Ren, J.; Chen, H.; Xu, B.; Song, N.; Li, H.; Liang, A.; Shen, G. Untargeted LC-MS-based metabonomics revealed that aristolochic acid I induces testicular toxicity by inhibiting amino acids metabolism, glucose metabolism, beta-oxidation of fatty acids and the TCA cycle in male mice. Toxicol. Appl. Pharmacol. 2019, 373, 26-38. [CrossRef]

40. Hertz, L.; Song, D.; Peng, L.; Chen, Y. Multifactorial Effects on Different Types of Brain Cells Contribute to Ammonia Toxicity. Neurochem. Res. 2017, 42, 721-736. [CrossRef]

41. Martínek, V.; Sklenár, J.; Dracínsky, M.; Sulc, M.; Hofbauerová, K.; Bezouska, K.; Frei, E.; Stiborová, M. Glycosylation protects proteins against free radicals generated from toxic xenobiotics. Toxicol. Sci. 2010, 117, 359-374. [CrossRef]

42. Wang, Z.; He, B.; Liu, Y.; Huo, M.; Fu, W.; Yang, C.; Wei, J.; Abliz, Z. In situ metabolomics in nephrotoxicity of aristolochic acids based on air flow-assisted desorption electrospray ionization mass spectrometry imaging. Acta Pharm. Sin. B. 2020, 10, 1083-1093. [CrossRef] [PubMed]

43. Zhao, Y.Y.; Wang, H.L.; Cheng, X.L.; Wei, F.; Bai, X.; Lin, R.C.; Vaziri, N.D. Metabolomics analysis reveals the association between lipid abnormalities and oxidative stress, inflammation, fibrosis, and Nrf2 dysfunction in aristolochic acid-induced nephropathy. Sci. Rep. 2015, 7, 12936. [CrossRef] [PubMed]

44. Klein, J. Membrane breakdown in acute and chronic neurodegeneration: Focus on choline-containing phospholipids. J. Neural. Transm. 2000, 107, 1027-1063. [CrossRef] [PubMed]

45. Bonvallot, N.; Tremblay-Franco, M.; Chevrier, C.; Canlet, C.; Warembourg, C.; Cravedi, J.P.; Cordier, S. Metabolomics tools for describing complex pesticide exposure in pregnant women in Brittany (France). PLoS ONE 2013, 8, e64433.

46. Zhang, Y.X.; Yang, X.; Zou, P.; Du, P.F.; Wang, J.; Jin, F.; Jin, M.J.; She, Y.X. Nonylphenol Toxicity Evaluation and Discovery of Biomarkers in Rat Urine by a Metabolomics Strategy through HPLC-QTOF-MS. Int. J. Environ. Res. Public Health 2016, 13, 501. [CrossRef] [PubMed] 
47. Pozdzik, A.A.; Salmon, I.J.; Debelle, F.D.; Decaestecker, C.; Van den Branden, C.; Verbeelen, D.; Deschodt-Lanckman, M.M.; Vanherweghem, J.L.; Nortier, J.L. Aristolochic acid induces proximal tubule apoptosis and epithelial to mesenchymal transformation. Kidney Int. 2008, 73, 595-607. [CrossRef]

48. Ni, Y.; Su, M.; Qiu, Y.; Chen, M.; Liu, Y.; Zhao, A.; Jia, W. Metabolic profiling using combined GC-MS and LC-MS provides a systems understanding of aristolochic acid-induced nephrotoxicity in rat. FEBS Lett. 2007, 581, 707-711. [CrossRef]

49. Miura, K.; Ishii, T.; Sugita, Y.; Bannai, S. Cystine uptake and glutathione level in endothelial cells exposed to oxidative stress. Am. J. Physiol. 1992, 262, C50-C58. [CrossRef]

50. Koppula, P.; Zhuang, L.; Gan, B. Cystine transporter SLC7A11/xCT in cancer: Ferroptosis, nutrient dependency, and cancer therapy. Protein Cell 2021, 12, 599-620. [CrossRef] 